OPEN ACCESS

Edited by:

Satyajit Rath,

National Institute of Immunology,

India

Reviewed by:

Shailaja Sopory,

Translational Health Science and

Technology Institute, India

Juan Carlos Salazar,

Connecticut Children's Medical

Center, United States

*Correspondence:

Xuemei Hu

xue-mei-hu@163.com

these authors have contributed equally to this work.

Specialty section:

This article was submitted

to Inflammation,

a section of the journal

Frontiers in Immunology

Received: 28 May 2017 Accepted: 07 August 2017

Published: 24 August 2017

Citation:

Li Z, Zhao M, Li T, Zheng J, Liu X, Jiang Y, Zhang $H$ and $H u X(2017)$ Decidual Macrophage Functional

Polarization during Abnormal

Pregnancy due to Toxoplasma gondii:

Role for LILRB4.

Front. Immunol. 8:1013.

doi: 10.3389/fimmu.2017.01013

\section{Decidual Macrophage Functional Polarization during Abnormal Pregnancy due to Toxoplasma gondii: Role for LILRB4}

\author{
Zhidan $\mathrm{Li}^{1+}$, Mingdong Zhao ${ }^{2 t}$, Teng $\mathrm{Li}^{1+}$, Jing Zheng ${ }^{3}$, Xianbing Liu', Yuzhu Jiang', \\ Haixia Zhang ${ }^{1}$ and Xuemei $\mathrm{Hu}^{1 *}$ \\ ${ }^{1}$ Department of Immunology, Medicine \& Pharmacy Research Center, Binzhou Medical University, Yantai, China, \\ ${ }^{2}$ Department of Radiology, Binzhou Affiliated Hospital of Binzhou Medical University, Binzhou, China, ${ }^{3}$ Department of \\ Gynecology and Obstetrics, Yantai Traditional Chinese Medicine Hospital, Yantai, China
}

During gestation, Toxoplasma gondii infection produces a series of complications including stillbirths, abortions, and congenital malformations. The inhibitory receptor, LILRB4, which is mainly expressed by professional antigen-presenting cells (especially macrophages and dendritic cells) may play an important immune-regulatory role at the maternal-fetal interface. To assess the role of LILRB4 during T. gondii infection, LILRB4 ${ }^{-/-}$ and T. gondii infected pregnant mouse models were established. Further, human primary-decidual macrophages were treated with anti-LILRB4 neutralizing antibody and then infected with T. gondii. These in vivo and in vitro models were used to explore the role of LILRB4 in T. gondii-mediated abnormal pregnancy outcomes. The results showed that abnormal pregnancy outcomes were more prevalent in LILRB4 ${ }^{-/}$infected pregnant mice than in wild-type infected pregnant mice. In subsequent experiments, expression levels of LILRB4, M1, and M2 membrane-functional molecules, arginine metabolic enzymes, and related cytokines were assessed in uninfected, infected, LILRB4-neutralized infected, and LILRB4 ${ }^{-/}$infected models. The results demonstrated T. gondii infection to downregulate LILRB4 on decidual macrophages, which strengthened M1 activation functions and weakened M2 tolerance functions by changing M1 and M2 membrane molecule expression, synthesis of arginine metabolic enzymes, and cytokine secretion profiles. These changes contributed to abnormal pregnancy outcomes. The results of this study provide not only a deeper understanding of the immune mechanisms operational during abnormal pregnancy, induced by T. gondii infection, but also identify potential avenues for therapeutic and preventive treatment of congenital toxoplasmosis.

Keywords: inhibitory receptor, LILRB4, Toxoplasma gondii, abnormal pregnancy outcomes, decidual macrophage

\section{INTRODUCTION}

Toxoplasma is one of the TORCHES (toxoplasmosis, rubella, cytomegalovirus, herpes simplex, syphilis). It is an obligate, intracellular, protozoan parasite that is widespread in nature and a frequent human pathogen (1). T. gondii infection is initially asymptomatic, but immune alterations accompany infection, and these result in adverse outcomes. In particular, infections 
during early pregnancy can result in premature births, miscarriages, anencephaly, and other adverse pregnancy outcomes $(2,3)$. The mechanism by which infection with $T$. gondii contributes to these adverse pregnancy outcomes is unknown.

Like a successful allograft, a specialized immune microenvironment is crucial to the maintenance of normal pregnancy (4). The microenvironment contains decidual immunological cell populations including macrophages, natural killer cells (NK), T regulatory cells (Treg), and dendritic cells (DC), which secrete immune-suppressive cytokines such as interleukin 10 (IL-10) and transforming growth factor beta $(5,6)$. Macrophages comprise $20-25 \%$ of these decidual immunological cell populations. These macrophages maintain some relatively stable functional molecules and cytokine expression profile, permitting maternal-fetal tolerance throughout gestation $(7,8)$. Macrophages are generally categorized as classically activated M1 type or alternatively activated M2 type macrophages $(9,10)$. In normal pregnancy, the M2 type is the major decidual macrophage population and these cells express the surface markers CD163, CD209, and 206. These macrophages secrete IL-10, which promotes immune tolerance, and also synthesize the arginine metabolic enzyme type-I arginase (Arg-I), which is involved in tissue remolding (11). In contrast, M1 type decidual macrophages have high levels of CD80 and CD86 expression. These macrophages secrete tumor necrosis factor alpha (TNF- $\alpha)$ and synthesize inducible nitric oxide synthase (iNOS), which promote a pro-inflammatory response (12). In mice, M2 decidual macrophages are characterized by expression of CD206 ${ }^{\text {high }}$, IL-10 ${ }^{\text {high }}$, and Arg-I ${ }^{\text {high }}$, whereas M1 decidual macrophages are characterized by expression of $\mathrm{CD} 80^{\text {high }}, \mathrm{CD} 86^{\text {high }}$, TNF- $\alpha^{\text {high }}$, and iNOS ${ }^{\text {high }}(12)$.

Human leukocyte immunoglobulin-like receptor subfamily B member 4 (LILRB4), or gp49B (in mice), is highly expressed on macrophages and is a member of the immunoglobulin-inhibitory receptor superfamily. LILRB4 contains putative immune receptor, tyrosine-based, inhibitory motifs, which recruit inhibitory phosphatases, transducing negative signals within cells $(13,14)$. High expression levels of LILRB4 are crucial for immune tolerance and immune regulation during normal pregnancy $(15,16)$. Acting as an endogenous negative regulator of macrophage activation, LILRB4 ligation reportedly inhibits TNF- $\alpha$ production, which modulates decidual macrophage function in mice (17).

Studies have reported that LILRB $4^{-/-}$mice were born at expected ratios, were healthy and fertile, and displayed normal long-term survival rates (18). It is unknown whether decidual macrophage LILRB4 is involved in abnormal pregnancy outcomes due to T. gondii. In this study, LILRB4 ${ }^{-/}$pregnant mice were infected with $T$. gondii, and the role of macrophage LILRB4 in abnormal pregnancy was assessed. Further, human decidual macrophages were infected with $T$. gondii in order to evaluate the potential involvement of LILRB4 in adverse human pregnancy outcomes.

\section{MATERIALS AND METHODS}

\section{Ethics Statement}

Sample collection procedures for this study were approved by the Binzhou Medical University Ethics Committee (Shandong,
China). All subjects provided written informed consent for the collection of samples and subsequent analysis. The Ethics Committees approved this consent procedure. This study was carried out in strict accordance with the recommendations in the Guide for the Care and Use of Laboratory Animals of Binzhou Medical University. The protocol was approved by the Committee on the Ethics of Animal Experiments of Binzhou Medical University. All procedures were performed under sodium pentobarbital anesthesia, and all efforts were made to minimize suffering of the animals.

\section{Animal Models}

C57BL/6 wild-type mice were purchased from Beijing Vital River Laboratory Animal Technology Co., Ltd. (Beijing, China). LILRB4-deficient (LILRB44 ${ }^{--}$) C57BL/6 mice were obtained from Riken BioResource Center (Tsukuba, Japan). Subsequently, 6- to 8-week-old female mice were housed five per cage, and 8- to 10-week-old male mice were housed one per cage. All mice were maintained in the specific pathogen-free animal house of Binzhou Medical University at $22-26^{\circ} \mathrm{C}$ with $50-60 \%$ humidity and a $12 \mathrm{~h}$ light/ $12 \mathrm{~h}$ dark cycle, with abundant sterilized water and food (Jiangsu Biological Engineering Co., Ltd., China). One day after cohabitation with males (at a ratio of 2 females: 1 male), female mice with vaginal plugs [gestational day (gd) 0] were segregated and randomized into uninfected, infected with T. gondii, and LILRB $4^{-/-}$infected with T. gondii groups.

\section{Genotyping of LILRB4-/- Mice}

Genomic DNA was extracted from mouse tails using a tissue DNA extraction kits (Generay, China). Polymerase chain reaction (PCR) was used to synthesize cDNA. After initial denaturation $\left(3 \mathrm{~min}\right.$ at $\left.95^{\circ} \mathrm{C}\right), \mathrm{PCR}$ was performed with 35 amplification cycles of denaturation for $30 \mathrm{~s}$ at $95^{\circ} \mathrm{C}$, annealing for $30 \mathrm{~s}$ at $55^{\circ} \mathrm{C}$, and extension for $60 \mathrm{~s}$ at $72^{\circ} \mathrm{C}$, followed by a final extension for $5 \mathrm{~min}$ at $72^{\circ} \mathrm{C}$. PCR products were separated by electrophoresis in $2 \%$ agarose gels, and sizes were estimated using Trans DNA marker I (100-700 bp; Transgene, France). Gels were stained with GelStain (10,000×; Transgene, France) to visualize DNA. Primers for PCR amplification were LILRB4 P1, 5'-ACCGGTGGATGTGGAATGTGTG-3'; LILRB4 P2, 5'-GTCCTGGGTTCCAGAATAAGAC-3'; and LILRB4 P3, 5'-TCTGCTCTTAGGAAATTACAGAA- ${ }^{\prime}$.

The expected PCR product sizes were 260 bp (mutant), 371 and $260 \mathrm{bp}$ (heterozygote), and $371 \mathrm{bp}$ (wild-type). Homozygous LILRB $4^{-/-}$mice were continually bred for the duration of the study.

\section{Preparation of $T$. gondii RH Tachyzoites}

Toxoplasma gondii tachyzoites were maintained in HEp-2 cells in minimum essential media (MEM) (Hyclone, USA), 5\% fetal bovine serum (FBS; Gibco, USA), and $100 \mathrm{IU} / \mathrm{ml}$ penicillin/ streptomycin, which were purchased from Sigma-Aldrich (USA). After culture, tachyzoites were centrifuged at $1,500 \mathrm{rpm}(433 \times g)$ for $10 \mathrm{~min}$, and purified tachyzoites were resuspended in MEM and counted using a Neubauer chamber. 


\section{Infection Models and Pregnancy Outcomes Assessment}

Pregnant mice (either LILRB44 ${ }^{--}$infected or wide-type infected groups of mice) were inoculated intraperitoneally (i.p.) with 400 tachyzoites in $200 \mu \mathrm{l}$ of sterile phosphate buffer (PBS) on gd 8. Uninfected groups of mice were inoculated with $200 \mu \mathrm{l}$ of sterile PBS. All mice were sacrificed at 6 days post-infection (dpi), uteri were removed, and the total number of implantation and resorption sites counted. Resorption sites were identified by their small size and the necrotic and hemorrhagic appearance of the embryos. Placenta were compared with those of uninfected mice. Abortion rates were calculated as the ratio of resorption sites to total implantation sites (resorptions plus normal implantation sites).

\section{Cell Preparation}

Embryos and placenta were dissected and removed from mice at gd 14. Dispersed cells were prepared from small pieces of placenta and uterine tissue using a GentleMACS dissociator (Miltenyi, Germany). Single cell suspensions were then obtained by filtration through $48 \mu \mathrm{m}$ sterile nets. After Ficoll density gradient centrifugation in mouse lymphocyte separation medium (TBD Science, China), mononuclear cells were collected from the white film layer and assessed by flow cytometry.

\section{Human Clinical Sample Collection}

Decidual tissues were taken from women without evidence of clinical genital infections during pregnancy. All voluntary abortions occurred in the Department of Obstetrics and Gynecology, Yantai Hospital of Traditional Chinese Medicine and Yantai Affiliated Hospital of Binzhou Medical University between 8 and 10 weeks after conception. Sample collection protocols were approved by the ethics Committee of Binzhou Medical University. Tissues were rinsed with sterile saline solution 5-8 times, and decidual tissues were picked and cultured in Dulbecco's modified eagle medium/high glucose medium (Hyclone, USA) supplemented with $100 \mathrm{IU} / \mathrm{ml}$ penicillin and $100 \mathrm{IU} / \mathrm{ml}$ streptomycin (Sigma-Aldrich, USA).

\section{Isolation of Human Decidual Macrophages}

Decidual tissues were immediately washed 5-6 times in Roswell Park Memorial Institute (RPMI) medium, cut into pieces, and then digested in $0.1 \%$ collagenase type IV (Sigma-Aldrich, USA) solution containing $25 \mathrm{IU} / \mathrm{ml}$ DNase-I (Sigma-Aldrich, USA) at $37^{\circ} \mathrm{C}$ for $30 \mathrm{~min}$. Single cell suspensions were obtained using a GentleMACS dissociator (Miltenyi Biotech, Germany) and were filtered through $48 \mu \mathrm{m}$ nylon mesh filters. Density gradient centrifugation was then performed using human lymphocyte separation medium (TBD Science, China) at 2,000 $\mathrm{rpm}(771 \times g)$ for $20 \mathrm{~min}$ at $20^{\circ} \mathrm{C}$ according to the manufacturer's instructions for mononuclear cell collection. Decidual macrophages were then purified using a human CD14 positive selection kit (Stem Cell Science, USA) according to the manufacturer's instructions with purity of $>95 \%$ for all experiments. Human decidual macrophages were counted, and aliquots containing more than $1.5 \times 10^{6}$ cells were allocated to uninfected, infected, and LILRB4-neutralized infected groups. Cells of the LILRB4-neutralized infected group were treated with neutralizing anti-LILRB4 antibody $(10 \mu \mathrm{g} /$ $\mathrm{mL}$ ) for $1 \mathrm{~h}$ prior to $T$. gondii infection, which was performed at a ratio of 2:1, T. gondii to decidual macrophages. Samples containing approximately $5.0 \times 10^{5}$ decidual macrophages were cultured in RPMI medium supplemented with $10 \%$ FBS (Gibco, USA), and $100 \mathrm{IU} / \mathrm{ml}$ penicillin and $100 \mathrm{IU} / \mathrm{ml}$ streptomycin (Sigma-Aldrich, USA) for $24 \mathrm{~h}$ at $37^{\circ} \mathrm{C}$ in a humidified $5 \% \mathrm{CO}_{2}$ incubator.

\section{Flow Cytometry}

The following fluorochrome-conjugated, mouse-specific monoclonal antibodies (mAbs) were used for assessment: Pe-cy7conjugated anti-F4/80 (marker of mouse-decidual macrophage), PE-conjugated anti-LILRB4, FITC-conjugated anti-CD206, FITC-conjugated anti-CD86, FITC-conjugated anti-CD80, APC-conjugated anti-TNF- $\alpha$ (all from BioLegend, USA), APCconjugated anti-IL-10 (Becton Dickinson, BD, USA), APCconjugated anti-iNOS (eBioscience, USA), and APC-conjugated anti-Arg-I (RD, USA). Isolated mononuclear cells were incubated with anti-F4/80, anti-LILRB4, and anti-CD206; or anti-F4/80, anti-LILRB4, and anti-CD80; or anti-F4/80, anti-LILRB4, and anti-CD86 $\mathrm{mAbs}$ at $4^{\circ} \mathrm{C}$ in the dark for $30 \mathrm{~min}$ and were then washed twice. To stain intracellular Arg-I and iNOS, cells were first incubated with anti-F4/80 and anti-LILRB $4 \mathrm{mAbs}$ at $4^{\circ} \mathrm{C}$ in the dark for $30 \mathrm{~min}$ and then washed. Subsequently, cells were fixed and permeabilized in 1× Fix/Perm buffer (eBioscience, USA) for 30 min according to the manufacturer's instructions. After washing twice, cells were incubated with anti-Arg-I and anti-iNOS $\mathrm{mAbs}$ at $4^{\circ} \mathrm{C}$ in the dark for $45 \mathrm{~min}$ and were then washed twice. For analysis of the intracellular cytokines IL-10 and TNF- $\alpha$, cells were initially stimulated for $4 \mathrm{~h}$ with leukocyte activation cocktail, with BD GolgiPlug (BD, USA). Cells were then collected and incubated with anti-F4/80 and anti-LILRB4 $\mathrm{mAbs}$ at $4^{\circ} \mathrm{C}$ in the dark for $30 \mathrm{~min}$ and then washed. The cells were then fixed and permeabilized in $1 \times$ Fix/Perm buffer (eBioscience, USA) at $4^{\circ} \mathrm{C}$ for $30 \mathrm{~min}$ according to the manufacturer's instructions. After washing twice, cells were incubated with anti-IL-10 or anti-TNF- $\alpha$ mAbs at $4^{\circ} \mathrm{C}$ in the dark for $45 \mathrm{~min}$ and washed.

Assays were performed with the following fluorochromeconjugated, human-specific mAbs: Pe-cy7-conjugated antiCD14, APC-conjugated anti-LILRB4, and PE-conjugated anti-TNF- $\alpha$, all from eBioscience, USA. FITC-conjugated anti-CD206, FITC-conjugated anti-CD163, FITC-conjugated anti-CD209, PE-conjugated anti-CD80, PE-conjugated antiCD86, and PE-conjugated anti-IL-10 were from BD, USA. Similarly, decidual macrophages were incubated with anti-CD14, anti-LILRB4, and anti-CD206; or anti-CD14, anti-LILRB4, and anti-209, or anti-CD14, anti-LILRB4, and anti-CD163, or antiCD14, anti-LILRB4, and anti-CD80, or anti-CD14, anti-LILRB4, and anti-CD86 $\mathrm{mAbs}$ at $4^{\circ} \mathrm{C}$ in the dark for $30 \mathrm{~min}$ and were then washed once. Decidual macrophages were also collected and incubated with anti-CD14, anti-LILRB4, and anti-IL-10, or anti-TNF- $\alpha$ mAbs (as described above for intracellular cytokine staining). Analysis was performed with a FACScanto ${ }^{\mathrm{TM}} \mathrm{II}$ instrument (Becton Dickinson, USA). 


\section{Enzyme-Linked Immunosorbent Assays (ELISA)}

Purified human decidual macrophages from uninfected, infected, and LILRB4-neutralized infected groups were cultured for $24 \mathrm{~h}$ after $T$. gondii infection, at a ratio of $T$. gondii to macrophage of $2: 1$. Cells were harvested from suspensions, and IL-10 and TNF- $\alpha$ levels were analyzed by ELISA according to the manufacturer's protocols. Standard curves were generated using standards for each assay, and all measurements of absorbance were performed in triplicate at $450 \mathrm{~nm}$. Concentrations were calculated according to standard curves and respective formulas.

\section{Western Blot}

Purified human decidual macrophages were harvested after treatment and washed with PBS, then lysed for 40 min on ice in $100 \mu \mathrm{l}$ of lysis buffer containing phenylmethanesulfonyl fluoride at 16:1 (Beyotime Biotech, China). Cell lysates were centrifuged at $12,000 \times g$ for $20 \mathrm{~min}$ at $4^{\circ} \mathrm{C}$, and supernatants were mixed with $5 \times$ sodium dodecyl sulfate-polyacrylamide (SDS-PAGE) loading buffer and boiled for $5 \mathrm{~min}$. Aliquots containing $40 \mu \mathrm{g}$ of protein were separated on $12 \%$ (for detection of Arg-I) or $8 \%$ (for detection of iNOS) SDS-PAGE gels. Proteins were then transferred onto polyvinylidene fluoride membranes (Millipore, USA) and were blocked with 7\% non-fat dry milk in Tris-buffered saline containing $0.2 \%$ Tween-20 (TBS-T) at room temperature for at least $4 \mathrm{~h}$. Membranes were then incubated with rabbit anti-human arginase-I and iNOS antibodies (1:1,000 dilution; Abcam, USA) at $4^{\circ} \mathrm{C}$ overnight. Membranes were then washed four times in $1 \times$ TBS-T for $10 \mathrm{~min}$ and incubated with horseradish peroxidase conjugated goat anti-rabbit IgG secondary antibody (1:2,000 dilution; Protech, USA) at room temperature for $1 \mathrm{~h}$. After washing three times for $15 \mathrm{~min}$, proteins on membranes were visualized using an enhanced chemiluminescence kit (ECL; F. Hoffmann-La Roche Ltd., Switzerland) at various exposure times. GAPDH was detected using rabbit anti-GAPDH polyclonal antibody (Protech, USA) as a loading control.

\section{Pathology Assessments}

Mouse placentas and uteri were removed after treatments and were washed three times and fixed with $4 \%$ paraformaldehyde immediately. Tissues were then washed in running water and placed in a graded ethanol series of 30,50, and $70 \%$ and were then paraffin embedded using standard methods. Paraffin sections were stained with hematoxylin and eosin dye (H\&E; Shanghai Novland Co., Ltd., China) according to the manufacturer's instructions. Images of paraffin embedded sections were recorded at $20 \times$ magnification and are presented with $5 \mu \mathrm{m}$ scale bars.

\section{Immunofluorescence Imaging}

Purified, human decidual macrophages from uninfected, infected, and LILRB4-neutralized infected groups were cultured for $24 \mathrm{~h}$ after T. gondii infection and then fixed in $4 \%$ paraformaldehyde for $15 \mathrm{~min}$ and blocked with goat serum for $1 \mathrm{~h}$. Cells were then incubated overnight at $4^{\circ} \mathrm{C}$ with anti-LILRB4 (Santa Cruz, Germany) and anti-CD163 (BD, USA), or anti-LILRB4 and anti-CD86 (BD, USA) antibodies. DyLight 488-goat anti-rabbit IgG (Abbkine,
USA) was used as a secondary antibody for anti-LILRB4 antibody, and DyLight 649-goat anti-mouse IgG (Abbkine, USA) was used as a secondary antibody for anti-CD163 and anti-CD86. Cells were incubated with appropriate concentrations of secondary antibodies at $37^{\circ} \mathrm{C}$ for $1 \mathrm{~h}$ and were subsequently stained with the nucleic acid stain $4^{\prime}, 6$-diamidino-2-phenylindole for $15 \mathrm{~min}$. Finally, cells were observed using a laser confocal microscope (Leica, Germany).

\section{Data Analysis and Statistics}

Data are presented as means \pm SD. Statistical analyses were performed using the GraphPad prism 5 statistics software package. Differences were identified using unpaired $t$-tests and were considered significant when two-tailed $p$ values were less than 0.05 or very significant when two-tailed $p$ values were less than 0.01 .

\section{RESULTS}

\section{Animal Models and Abnormal Pregnancy Outcomes}

Infected pregnant mice were unkempt, had less mobility, erect fur, and placentas that were significantly inflamed with hyperemia. Absorbed fetuses and stillbirths were more prevalent in infected mice than in uninfected pregnant mice (Figures 1A,B). Infected mice had significantly lower placental and fetal weights as well as higher abnormal fetal ratios than uninfected mice (Figure 1D). Paraffin embedded sections of infected but not uninfected placentas showed damage as hemorrhage and lymphocyte infiltration, with spiral arteries (Figure 1E). In LILRB4 ${ }^{-/-}$infected mice, pregnancy outcomes were worse than outcomes observed in infected wild-type mice, which included more severe placental ischemia. Relative to wide-type infected mice, LILRB4 ${ }^{-/-}$infected fetuses were almost shapeless (Figures 1B,C), with reduced placental and fetal weights and abnormal fetal ratios (Figure 1D). Hemorrhage and lymphocyte infiltration were further exacerbated in the LILRB $4^{-1-}$ infected mice compared with infected wide-type mice (Figure 1E). Moreover, the percentage of decidual macrophages in all infected groups of mice was significantly higher than in the uninfected mice but did not differ significantly from that of the LILRB $^{-/-}$infected mice (Figure 1F).

\section{LILRB4 Expression by Decidual Macrophages Is Downregulated after T. gondii Infection}

By immunofluorescence (Figures 2A,B) and flow cytometry (Figures 2C,D), LILRB4 expression levels were significantly decreased on human decidual macrophages after T. gondii infection, in comparison with uninfected macrophages. As judged by flow cytometry, murine decidual macrophage levels of LILRB4 were downregulated in mice infected with T. gondii (Figures 2E,F).

\section{Expression of M1 and M2 Membrane Molecules Is Changed When LILRB4 Is Downregulated by T. gondii Infection of Decidual Macrophages}

Flow cytometry demonstrated LILRB4 to be significantly downregulated on human decidual macrophages by $T$. gondii infection. 


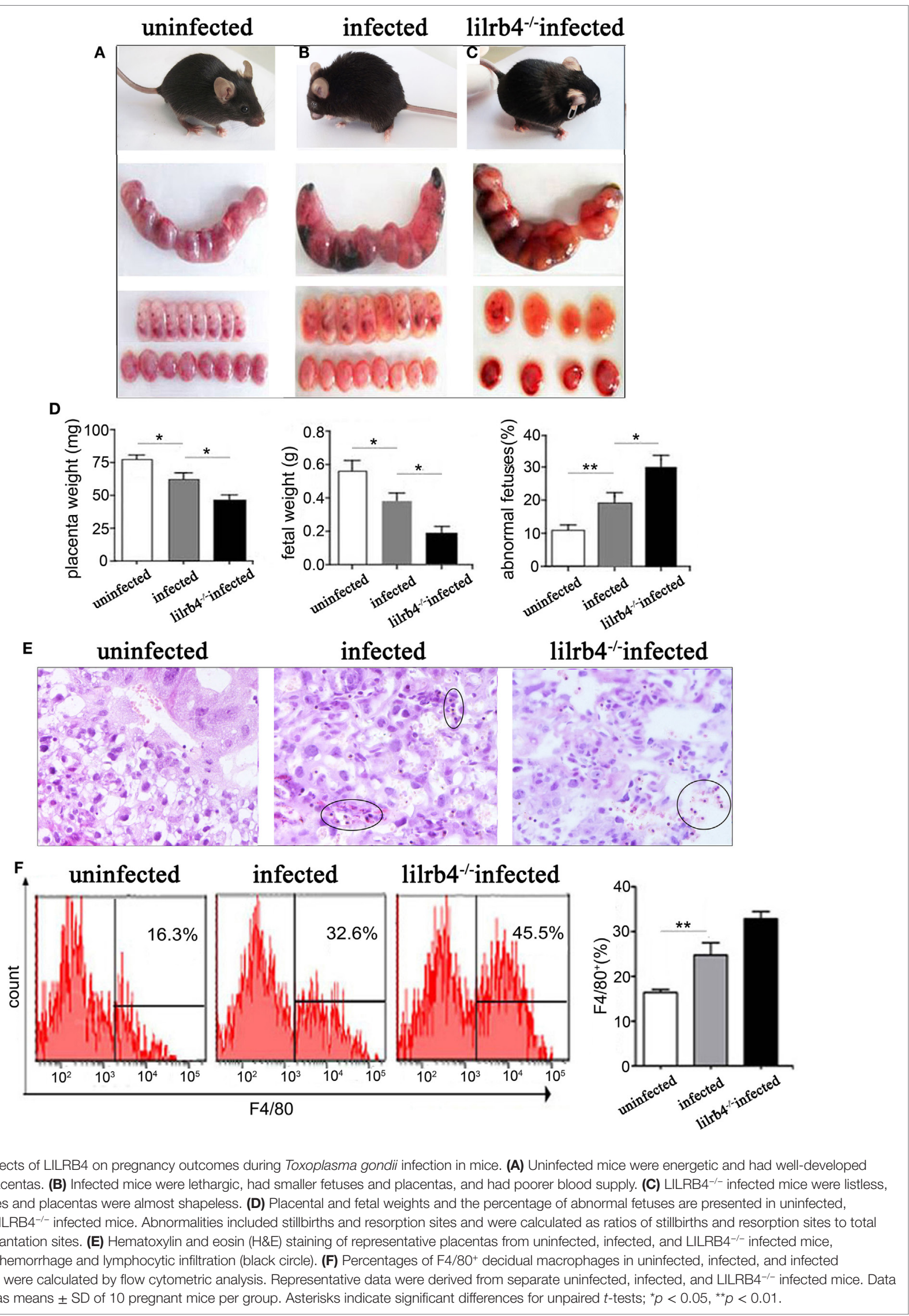



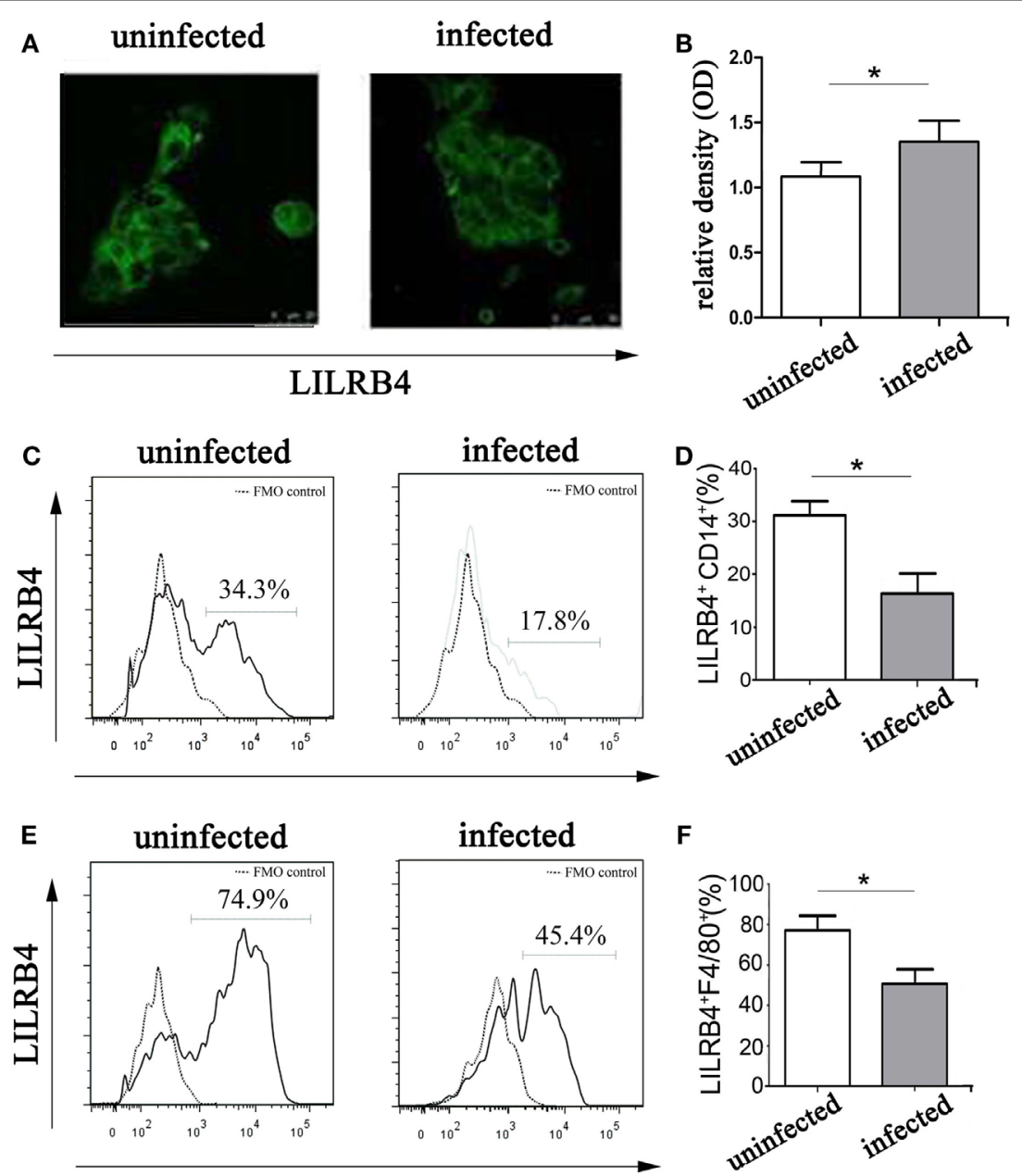

FIGURE 2 | Expression of LILRB4 on decidual macrophages infected with Toxoplasma gondii. (A) LILRB4 expression levels in uninfected and infected human decidual macrophages were compared by immunofluorescence. (B) Histogram analysis of immunofluorescence for LILRB4 expression levels in uninfected and infected human decidual macrophages. (C) LILRB4 expression levels in uninfected and infected human decidual macrophages were compared by flow cytometry analyses. The flow cytometric FMO as the control. (D) Histogram analysis of flow cytometry for LILRB4 expression levels in uninfected and infected human decidual macrophages. (E) Flow cytometric analysis of LILRB4 expression changes in uninfected and infected mouse-decidual macrophages. The flow cytometric FMO as the control. (F) Histogram analysis of flow cytometry for LILRB4 expression levels in uninfected and infected mouse-decidual macrophages. Representative data for in vitro analysis of one individual from the uninfected and the infected groups. Data are presented are means \pm SD ( $\left.{ }^{*} p<0.05\right)$ of 10 pregnant mice and 9 human spontaneous abortion specimens. Differences were identified by unpaired $t$-tests.

M1 membrane-functional molecules CD80 (Figures 3A,B) and CD86 (Figures 3C,D) were significantly upregulated. In macrophages in which LILRB4 was neutralized, M1 membranefunctional molecules were further upregulated relative to infected cells (Figures 3A-D). M2 membrane-functional molecules CD163, CD209, and CD206 were significantly downregulated on human decidual macrophages after $T$. gondii infection and were slightly increased in the LILRB4-neutralized macrophages compared with infected macrophages (Figures 3E,F for CD163, Figures 3G,H for CD209, and Figures 3I,J for CD206). Furthermore, M1/M2 ratios (CD80/CD163, CD80/CD209, CD80/CD206, CD86/CD163, CD86/CD209, and CD86/CD206) were lowest in uninfected, greater in infected, and greatest in LILRB4-neutralized and infected macrophages (Figure 3K).
By immunofluorescence, infected macrophages had decreased LILRB4 and CD163 and increased CD86 expression when compared with uninfected macrophages. In the infected, LILRB4-neutralized macrophages, LILRB4 expression level was very low, whereas CD163 was slightly upregulated and CD86 was significantly upregulated in comparison with infected macrophages (Figures 4A,B). Similar to these in vitro studies, M1 membrane-functional molecules CD80 and CD86 were both significantly increased after T. gondii infection and increased further in LILRB4 ${ }^{-/-}$infected mice (Figures 5A,D for CD80, Figures 5B,E for CD86). In contrast, the M2 membranefunctional molecule CD206 was significantly decreased after T. gondii infection and was slightly increased in LILRB $4^{-/}$infected mice when compared with infected mice (Figures 5C,F). CD80/ 

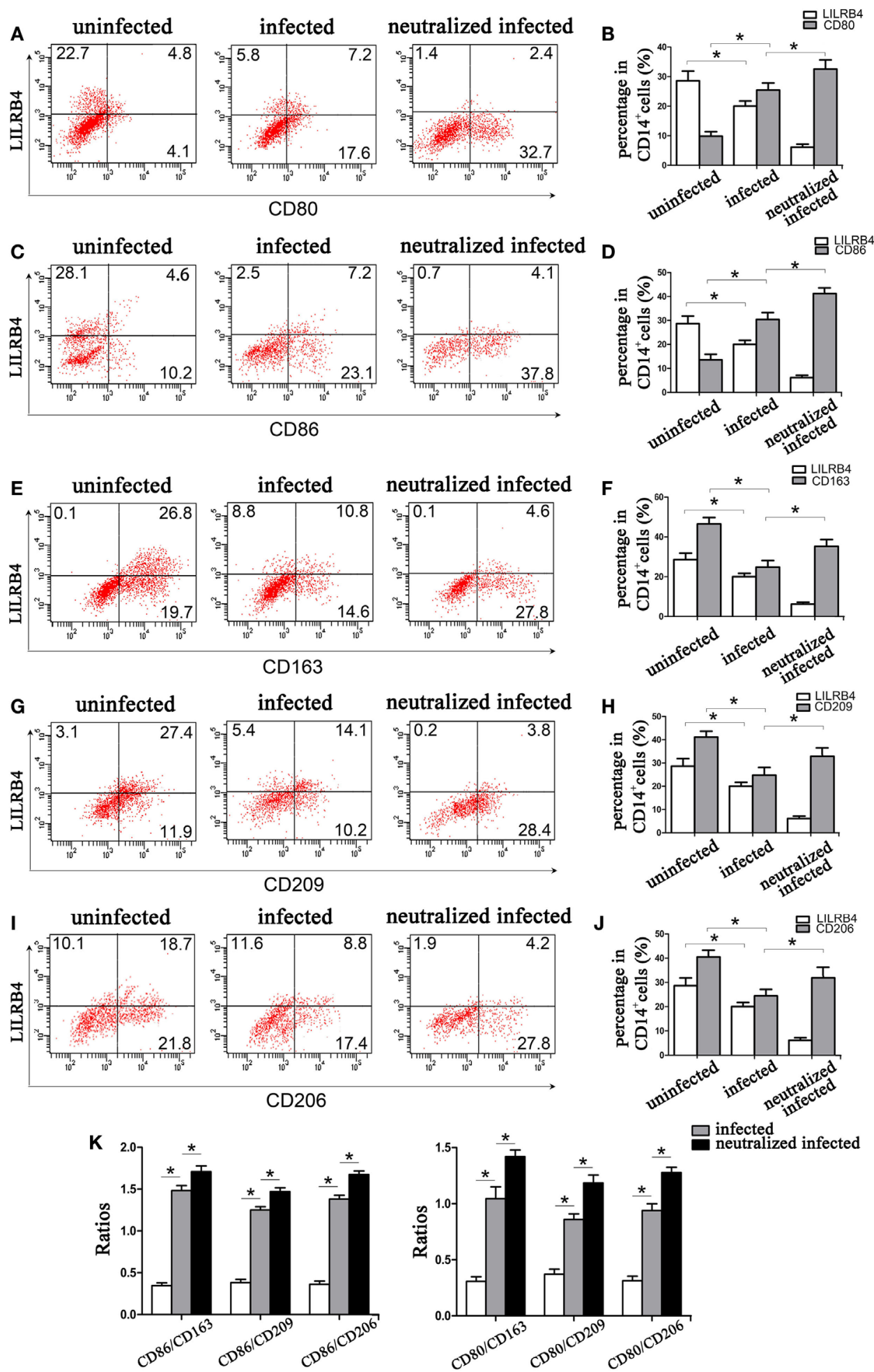

FIGURE 3 | Downregulation of LILRB4 on human decidual macrophages infected with Toxoplasma gondii. Changes in the expression of M1 and M2 membranefunctional molecules. Histograms of flow cytometry in uninfected, infected, and LILRB4-neutralized and infected human decidual macrophage are presented. Representative histograms of M2 membrane-functional molecules: CD163 (E,F), CD209 (G,H), and CD206 (I,J). Representative histograms of M1 membranefunctional molecules CD80 (A,B) and CD86 (C,D). (K) M1/M2 ratios (CD86/CD163, CD86/CD209, CD86/CD206, CD80/CD163, CD80/CD209, and CD80/CD206) for uninfected, infected, and LILRB4-neutralized and infected human decidual macrophages. Representative data were from one individual for uninfected, infected and LILRB4-neutralized and infected groups, respectively. Data are presented as means $\pm \mathrm{SD}\left({ }^{\star} p<0.05\right.$, $\left.{ }^{* *} p<0.01\right)$; nine human spontaneous abortion specimens were assayed for each group and were compared by unpaired $t$-tests. 


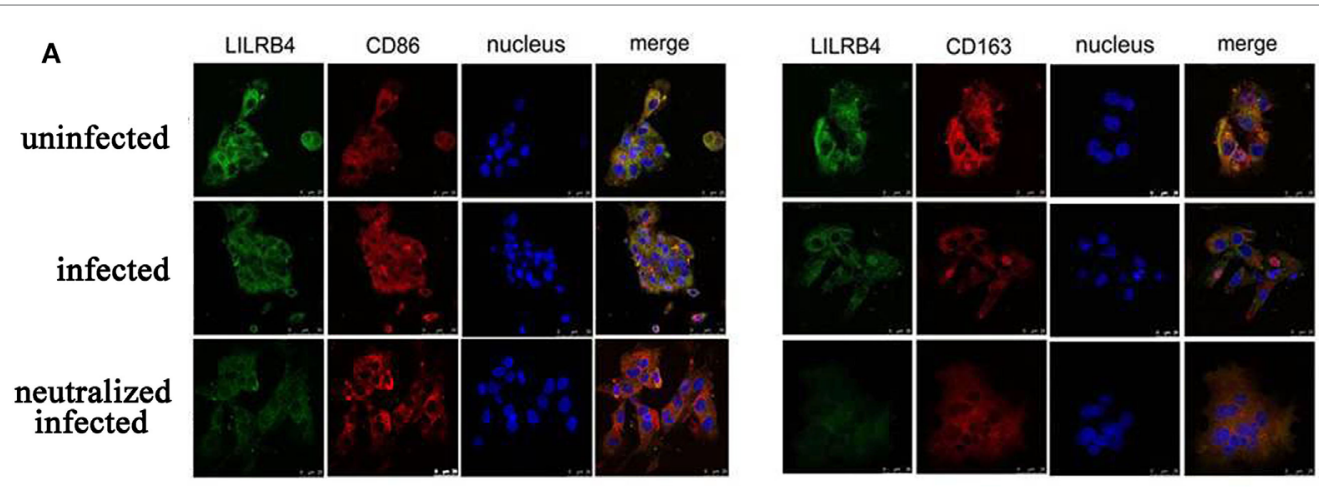

B

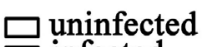

infected

neutralized infected
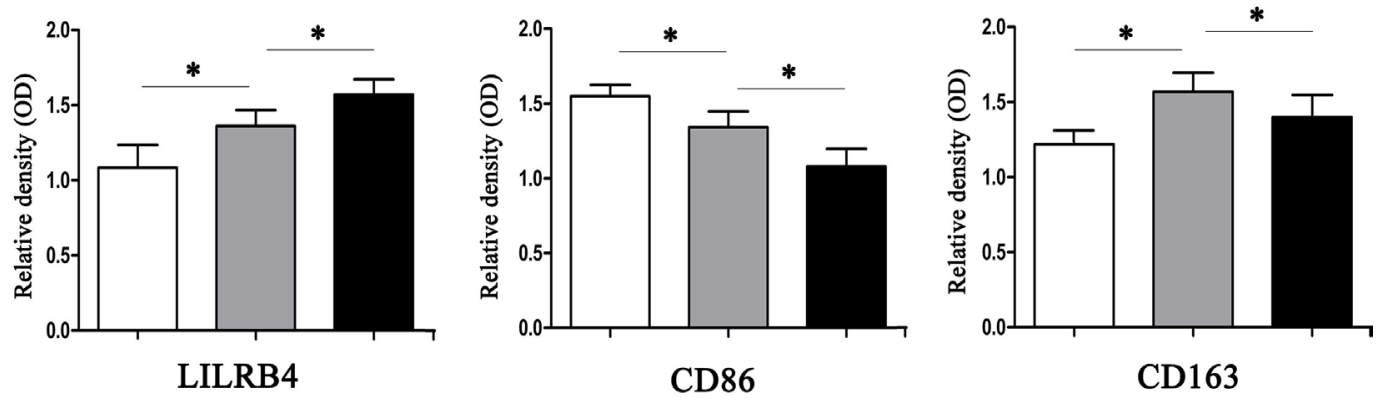

FIGURE 4 | Downregulation of LILRB4 on human decidual macrophages infected with Toxoplasma gondii. Changes in the expression of M1 and M2 membrane-functional molecules CD163 and CD86. (A) Representative immunofluorescent photographs of LILRB4 (green), CD163 (red), and CD86 (red) expression in uninfected, infected, and LILRB4-neutralized and infected human decidual macrophages. Nuclei (blue) were stained with 4',6-diamidino-2phenylindole. (B) Histograms analysis of LILRB4, CD163, and CD86 expression in uninfected, infected, and LILRB4-neutralized and infected human decidual macrophages. Representative data are for one individual from uninfected, infected, and infected LILRB4-neutralized groups, respectively. Data are presented as means $\pm \mathrm{SD}\left({ }^{*} p<0.05,{ }^{* *} p<0.01\right)$. Comparisons were by unpaired $t$-test; seven human spontaneous abortion specimens were assayed individually for each group.

CD206 and CD86/CD206 ratios were lowest in uninfected, greater in infected, and greatest in $\mathrm{LILRB}^{-/-}$infected mice (Figure 5G).

\section{LILRB4 Downregulation by T. gondii Infection of Decidual Macrophages Alters Expression of the Arginine Catabolism Enzymes Arg-I and iNOS}

By western blot analysis, the arginine catabolism enzyme iNOS was not detected in uninfected human decidual macrophages. After T. gondii infection the enzyme increased. The increase was even greater in infected LILRB4-neutralized macrophages. Arg-I synthesis in human decidual macrophages was significantly reduced following $T$. gondii infection and was further reduced in infected LILRB4-neutralized macrophages (Figures 6A,B). In vivo flow cytometric analysis demonstrated iNOS in decidual macrophages from T. gondii infected mice, with greater detection in decidual macrophages from infected LILRB $^{-1-}$ mice (Figures 6C,D). Arg-I expression was decreased in decidual macrophages from infected mice and further decreased in decidual macrophages from LILRB4 ${ }^{-/-}$infected mice (Figures 6E,F).

\section{Decidual Macrophage Secretion of TNF- $\alpha$ and IL-10 Is Affected When LILRB4 Is Downregulated by T. gondii Infection}

By flow cytometry, TNF- $\alpha$ levels within human decidual macrophages were increased following $T$. gondii infection and were further increased in infected LILRB4-neutralized cells (Figures 7A,C). IL-10 levels within human decidual macrophages were increased by $T$. gondii infection and reduced in infected LILRB4-neutralized cells (Figures 7 B,C). TNF- $\alpha /$ IL-10 ratios were greater in infected cells than in uninfected cells and were further increased in infected LILRB4-neutralized cells (Figure 7D). ELISA analysis showed increased TNF- $\alpha$ secretion in supernatants from infected human decidual macrophages, which was further increased in supernatants from infected LILRB4-neutralized cells. IL-10 in supernatants from human decidual macrophages was increased after T. gondii infection and decreased in supernatants from infected human LILRB4-neutralized macrophages, when compared with the infected cells (Figure 7E). TNF- $\alpha /$ IL-10 ratios were increased in infected cells compared with uninfected cells and were further increased in infected LILRB4-neutralized cells (Figure 7F) By flow cytometry, TNF- $\alpha$ was increased in decidual macrophages from infected mice and was further 

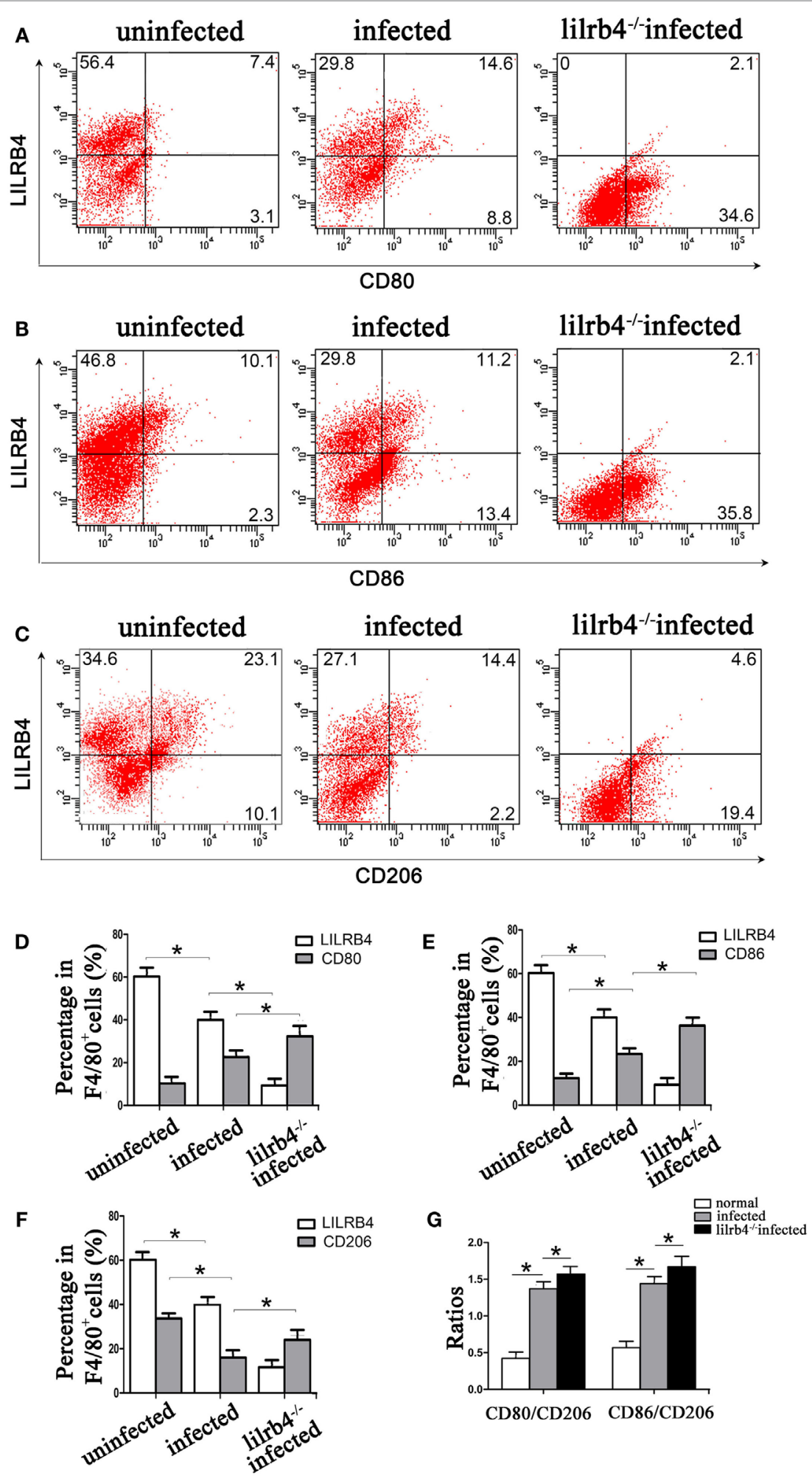

FIGURE 5 | Downregulation of LILRB4 in mouse-decidual macrophages by Toxoplasma gondii infection changed the expression of M1 and M2 membranefunctional molecules. Representative histograms of M2 membrane-functional molecules CD206 (C,F) and M1 membrane-functional molecules CD80 (A,D) and CD86 (B,E) for uninfected, infected, and LILRB4-/- infected mouse-decidual macrophage by flow cytometry. (G) Ratios of CD86/CD206 and CD80/CD206 in decidual macrophages from uninfected, infected, and LILRB4 ${ }^{-/}$infected mice. Representative data were from different individuals in uninfected, infected, and

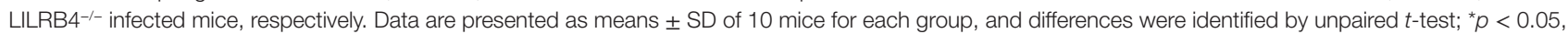
${ }^{\star \star} p<0.01$. 

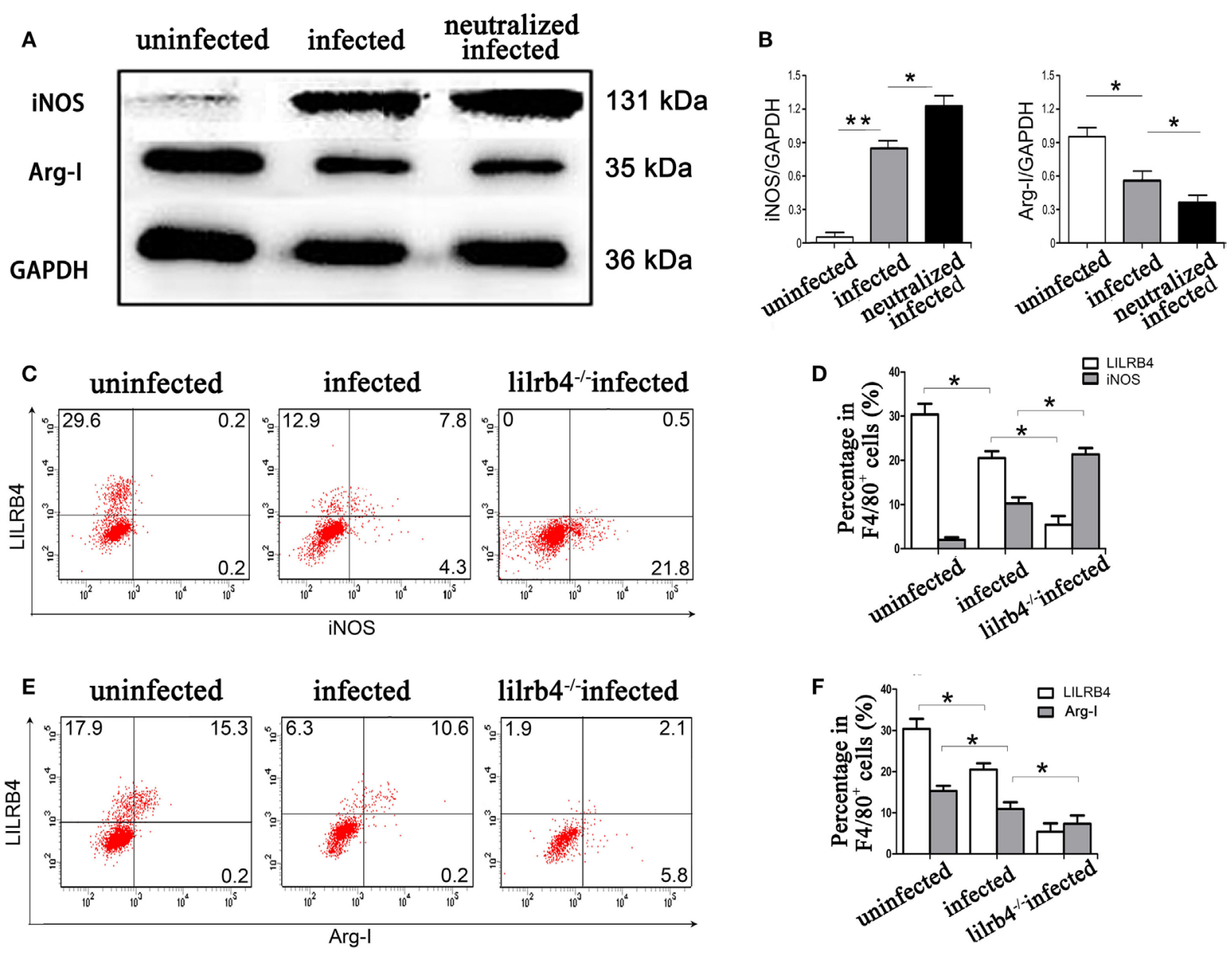

FIGURE 6 | Downregulation of LILRB4 on decidual macrophages by Toxoplasma gondii infection changed the expression of the arginine catabolism enzymes type-I arginase (Arg-I) and inducible nitric oxide synthase (iNOS). (A) Representative depictions of iNOS (131 kDa) and Arg-I (35 kDa) protein levels in uninfected, infected, and LILRB4-neutralized and infected human decidual macrophages by western blot. (B) Histograms analysis of western blot for iNOS and Arg-I expression in uninfected, infected, and LILRB4-neutralized and infected human decidual macrophages. Representative histograms for iNOS (C,D) and Arg-I (E,F) protein levels in uninfected, infected, and LILRB4 ${ }^{-/-}$infected mouse-decidual macrophages by flow cytometry. Representative data for in vivo analysis of different individuals in uninfected, infected, and LILRB4 ${ }^{-/}$infected groups. Representative data for in vitro analysis of one individual from uninfected, infected and LILRB4-neutralized and infected groups. Data are expressed as means \pm SD of 10 pregnant mice or 9 human spontaneous abortion samples for each treatment group; ${ }^{*} p<0.05$, ${ }^{* *} p<0.01$, by unpaired $t$-test.

increased in decidual macrophages from infected LILRB4 ${ }^{-1-}$ mice (Figures 8A,C). IL-10 secretion by mouse-decidual macrophages was increased by $T$. gondii infection, while IL-10 secretion was decreased in decidual macrophages from infected LILRB4 ${ }^{-1-}$ mice (Figures 8B,C). Finally, TNF- $\alpha / \mathrm{IL}-10$ ratios were increased in infected decidual macrophages compared with uninfected cells. A further increase was observed in decidual macrophages from infected LILRB4 ${ }^{-/}$mice (Figure 8D).

\section{DISCUSSION}

Toxoplasma gondii is a zoonotic parasite that causes toxoplasmosis. The greatest $T$. gondii hazard is vertical transmission during pregnancy $(1,19)$, with increased incidence of abortion, stillbirth, and congenital anencephaly (3, 20, 21). Successful pregnancy requires subtle regulation by immune cell populations and their secreted cytokines at the maternal-fetal interface (22). Studies have confirmed that during gestation, T. gondii disturbs the maternal-fetal interface, resulting in abnormal pregnancy outcomes $(3,21)$. Our previous work showed that functional molecules and cytokines produced by maternal NK, Treg, and DC cells are affected by $T$. gondii infection and that these cells contribute to abnormal pregnancy $(21,23)$. Decidual macrophages are a major subset of decidual immune cells and contribute to local immune tolerance during normal pregnancies $(24,25)$. LILRB4 is predominantly expressed on macrophages and is an inhibitory receptor that is central to immune tolerance (26). Specifically, LILRB4 acts as an endogenous negative regulator of macrophage activation, modulating cytokine secretion during pregnancy (15-17). However, it is unknown whether decidual macrophage LILRB4 participates in abnormal pregnancy outcomes during T. gondii infection. In this study, we observed high levels of 

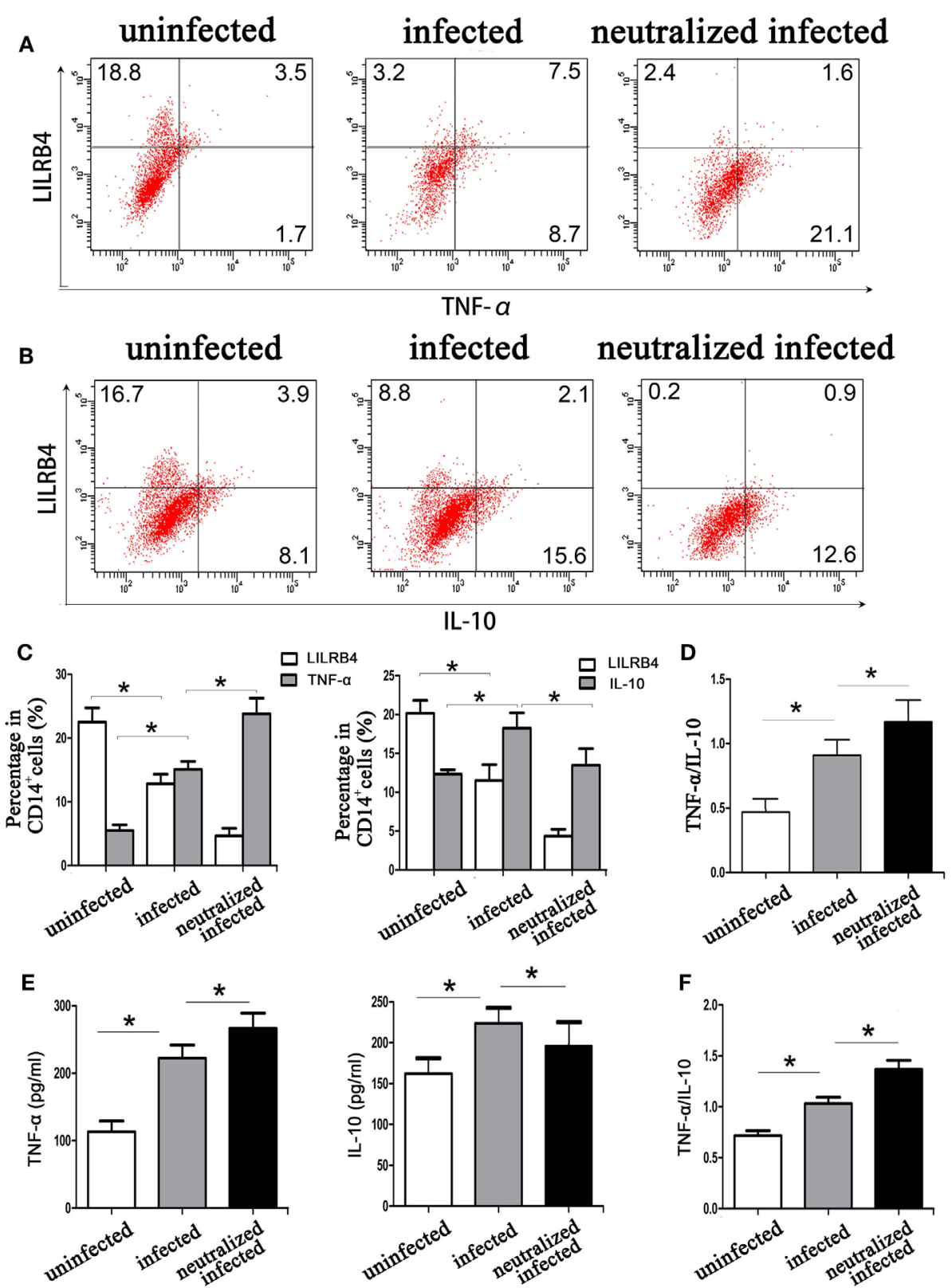

FIGURE 7 | Downregulation of LILRB4 on decidual macrophages by Toxoplasma gondii infection affects tumor necrosis factor alpha (TNF- $\alpha$ ) and interleukin 10 (IL-10) secretion by decidual macrophages. TNF- $\alpha$ (A) and IL-10 (B) levels in uninfected, infected, and LILRB4-neutralized and infected human decidual macrophages were assessed by flow cytometry analyses. (C) Histograms analysis for TNF- $\alpha$ and IL-10 in uninfected, infected, and LILRB4-neutralized infected human decidual macrophages (D) Ratios of TNF- $\alpha / \mathrm{IL}-10$ in uninfected, infected and LILRB4-neutralized, and infected human decidual macrophages. (E) IL-10 levels, TNF- $\alpha$ levels, and TNF- $\alpha /$ IL-10 ratios (F) were analyzed in supernatants from uninfected, infected, and LILRB4-neutralized and infected human decidual macrophages by enzyme-linked immunosorbent assays (ELISA). Representative data for in vitro analysis were from one individual in uninfected, infected, and LILRB4-neutralized and infected groups. ELISA samples were assayed in triplicate. Data are expressed as means \pm SD of nine human spontaneous abortion specimens from each group; ${ }^{*} p<0.05$ by unpaired $t$-test.

LILRB4 on uninfected mouse-decidual macrophages but low levels of LILRB4 in association with abnormal pregnancy outcomes mediated by $T$. gondii infection. To evaluate the relationship of LILRB4 to abnormal pregnancy outcomes, we established LILRB $^{-1-}$ and T. gondii infected pregnant mouse models and then compared pregnancy outcomes with those of T. gondii infected wild-type pregnant mice. Interestingly, pregnancy outcomes in infected LILRB4 ${ }^{-/}$pregnant mice were more severe and showed more stillbirths and absorbed sites, smaller placental and fetal size, and more severe hyperemia, when compared with wild-type infected mice. Compared with uninfected mice, T. gondii infected micehad a significant increase in decidual macrophages suggesting 

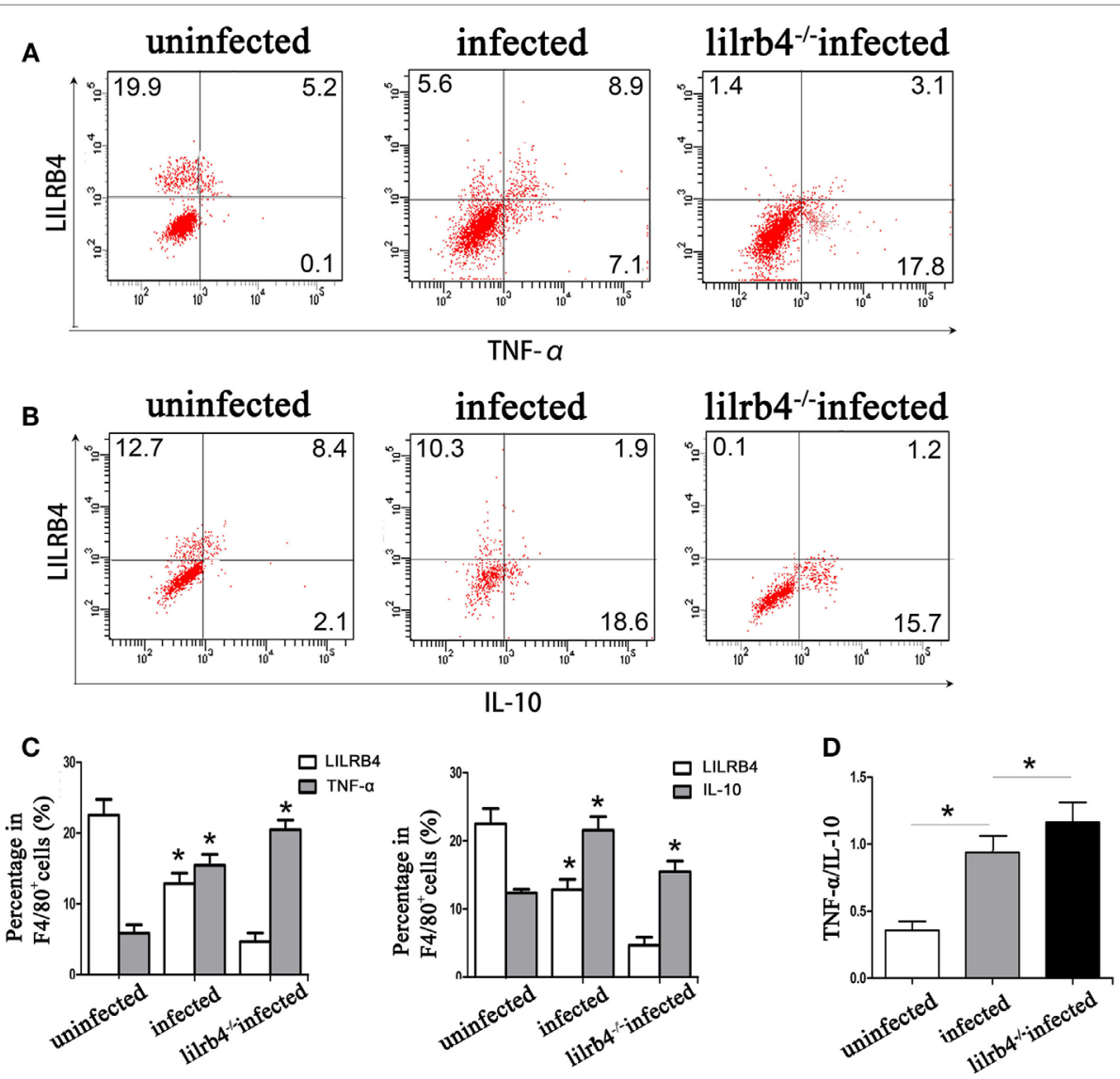

FIGURE 8 | Downregulation of LILRB4 on mouse-decidual macrophages with Toxoplasma gondii infections changed IL-10 and TNF- $\alpha$ secretion. TNF- $\alpha$ (A) and IL-10 (B) in decidual macrophages from uninfected, infected, and LILRB4 ${ }^{-/-}$infected were assessed by flow cytometry. (C) Histogram analysis of flow cytometry for

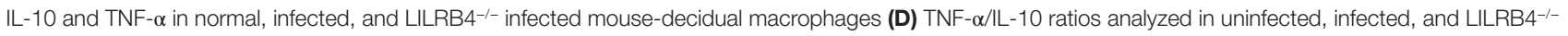
infected mice decidual macrophages. Representative data for in vivo analysis of different individuals from uninfected, infected, and LILRB4 ${ }^{-/}$infected groups. Data are expressed as means \pm SD of 10 animals from each group; ${ }^{*} p<0.05$ by unpaired $t$-test.

that infection induced a pro-inflammatory response. A modest increase in decidual macrophages between infected and LILRB $4^{-/-}$infected mice was not significant. These results suggest that LILRB4 plays a role during pregnancy, and that the role of LILRB4 is demonstrable during T. gondii infection.

LILRB4 expression was monitored on decidual macrophages during T. gondii infection both in vitro and in vivo. Those experiments showed LILRB4 expression to be significantly downregulated by $T$. gondii infection in both human and mouse-decidual macrophages during pregnancy. The prevalence of abnormal pregnancy outcomes among infected LILRB4 ${ }^{-/-}$mice further suggests that LILRB4 downregulation on decidual macrophages may play a role in the development of $T$. gondii-mediated abnormalities. To assess a mechanistic basis for these observations, we determined expression levels of functional molecules in decidual macrophages including membrane molecules, arginine metabolic enzymes, and cytokines, during infection.

Decidual macrophages are classified as M1 or M2 subsets (9, 10). M1 decidual macrophages are characterized by high expression of CD80 and CD86 and are associated with inflammatory responses and poor maternal-fetal tolerance (8). Excessive numbers of M1 decidual macrophages promote trophoblast apoptosis and retard placental development (12). In addition, upregulation of the costimulatory molecules CD80 and CD86 reportedly contributes to an abnormal immune microenvironment and a shift to a Th1 response at the maternal-fetal interface, resulting in human miscarriage (27). In contrast, M2 decidual macrophages express CD206, CD209, and CD163, and these macrophages mediate immunosuppression and maternal-fetal tolerance $(25,28)$. M2 decidual macrophage CD206 sustains immune homeostasis and immunosuppression at the maternalfetal interface (29). Similarly, CD209, mainly expressed on M2 decidual macrophages, contributes to maternal-fetal immune tolerance (30). CD163 is known as a homeostatic scavenger receptor and has been associated with tissue homeostasis and immune regulation $(11,25)$. T. gondii infection reportedly affects the polarization of macrophages during rat pregnancy, which further suggests that macrophage polarization is involved in abnormal pregnancy outcomes (31). Herein, we examined the effects of T. gondii on expression of M1 and M2 related membranefunctional molecules during pregnancy as well as in cultured human and mouse-decidual macrophages. Those experiments 
showed that with LILRB4 downregulation, the M1 membranefunctional molecules CD80 and CD86 were significantly upregulated, whereas the M2 membrane-functional molecules CD206, CD209, and CD163 were significantly downregulated both in vivo and in vitro. These data suggest that in addition to downregulating LILRB4, T. gondii infection contributes to the development of abnormal pregnancy outcomes by skewing decidual macrophages from an M2 type to an inflammatory M1 type. To clarify the role of LILRB4, we performed experiments in which LILRB4 was neutralized with antibody in vitro and in vivo by use of LILRB $4^{-1-}$ mice during T. gondii infection.

The M1 membrane-functional molecules CD80 and CD86 were upregulated in infected LILRB4-neutralized human decidual macrophages and in decidual macrophages from infected LILRB4 $^{-1-}$ mice. M2 membrane-functional molecules CD206, CD209, and CD163 were moderately upregulated as judged by flow cytometry. Immunofluorescence analysis of CD163, CD86, and LILRB4 on human decidual macrophages was consistent with the flow cytometric analysis. M1/M2 ratios for CD80/CD163, CD80/CD206, CD80/CD209, CD86/CD163, CD86/CD206, and CD86/CD209 suggested a tendency toward M1 functional molecules in LILRB4-neutralized and infected human decidual macrophages and in infected LILRB $4^{-/}$mice. Downregulation of LILRB4 on decidual macrophages during T. gondii infection likely shifts macrophages toward an M1 type and away from an M2 type, which likely contributes to abnormal pregnancy outcomes during T. gondii infection.

Decidual macrophages synthesize the arginine metabolic enzymes Arg-I and iNOS, which are associated with M1 and M2 phenotypes, respectively, and are widely used to distinguish between M1 and M2 macrophages $(32,33)$. Arg-I enhances the bioavailability of L-arginine, which is involved in immunosuppression and reportedly promotes polyamine synthesis to enhance placental growth and development $(34,35)$. iNOS in human placentas is absent under normal conditions, whereas excessive expression of iNOS suppresses placental vascular development $(36,37)$. In this study, results show induced expression of iNOS in M1 human and mouse-decidual macrophages after $T$. gondii infection, with no expression in uninfected cells. In contrast, Arg-I synthesis was downregulated in T. gondii-infected decidual macrophages in vivo and in vitro. Hence, along with LILRB4 downregulation, T. gondii-induced iNOS expression enhanced M1 decidual macrophage activation and downregulated Arg-I expression hampering immune suppression by M2 decidual macrophages. An anti-LILRB4 neutralizing antibody and LILRB $^{-1-}$ mice, infected with T. gondii, were used to determine whether changes in the arginine metabolic enzymes Arg-I and iNOS during T. gondii infection were a consequence of decreased LILRB4 expression. In those experiments, iNOS upregulation was observed in anti-LILRB4-neutralized and infected human decidual macrophages and in LILRB4 ${ }^{-1-}$ infected mice. In contrast, Arg-I expression was downregulated under these conditions. Hence, changes in Arg-I and iNOS expression during T. gondii infection are associated with decreased LILRB4 expression in decidual macrophages, which appears to relate to the prevalence of M1 decidual macrophages. An imbalance of M1 and M2 decidual macrophages, due to change in arginine metabolic enzymes, may contribute to the development of abnormal pregnancy outcomes during T. gondii infection.

Previously, IL-10 in decidual macrophage has been linked to the development of Th2-polarized local immunity. T-cell anergy to fetal antigens has been associated with the IL-10 regulatory and tolerant properties of decidual macrophages (29). IL-10 therapy of $T$. gondii infections reduces trophoblasts apoptosis at the maternal-fetal interface and improves pregnancy outcomes (38, 39). Decidual macrophage LILRB4 ligation by Fc $\gamma$ RI inhibited TNF- $\alpha$ production and hampered functional activation (17). Moreover, activated decidual macrophages can produce high levels of TNF- $\alpha$ at the maternal-fetal interface, and interactions with TNF- $\alpha$ receptors on extravillous trophoblasts induce trophoblast apoptosis in vitro $(40,41)$. The major cytokines TNF- $\alpha$ and IL-10 are secreted by decidual macrophages at the maternal-fetal interface and are involved in the balance of M1 and M2 phenotypes $(11,42)$. Herein, both TNF- $\alpha$ and IL-10 levels were increased in decidual macrophages. TNF- $\alpha / \mathrm{IL}-10$ ratios were increased after T. gondii infection of both human and mouse-decidual macrophages, suggesting a pro-inflammatory rather than an immunosuppressive environment. These data were confirmed by ELISA and by flow cytometry.

Finally, we determined whether changes in TNF- $\alpha$ and IL-10 secretion during $T$. gondii infection were due to a decrease in expression of LILRB4 by decidual macrophages. Both flow cytometry and ELISA analysis of supernatants showed increased TNF- $\alpha$ and decreased IL-10 expression and secretion levels in infected LILRB4 ${ }^{-/}$mice decidual macrophages and in infected human decidual macrophages that were treated with anti-LILRB4 neutralizing antibody. These data indicate that T. gondii-mediated reductions in LILRB4 result in the observed changes in TNF- $\alpha$ and IL-10 expression and suggest a prominent role for LILRB4 at the maternal-fetal interface. An imbalance in cytokines at the maternal-fetal interface likely contributes to the development of abnormal pregnancy outcomes during $T$. gondii infection.

Taken together, the results of this study show that downregulation of the inhibitory receptor LILRB4 on decidual macrophages during $T$. gondii infection strengthens an M1 activating and weakens an M2 tolerance response by altering M1 and M2 membrane molecule expression, the synthesis of arginine metabolic enzymes, and the cytokine secretory profile. These alterations result in abnormal pregnancy outcomes. This investigation contributes to an understanding of the immune mechanisms that result in abnormal pregnancy outcomes due to T. gondii infection.

\section{ETHICS STATEMENT}

Sample collection procedures for this study were approved by the Binzhou Medical University Ethics Committee (Shandong, China). All subjects provided written informed consent for the collection of samples and subsequent analysis. The Ethics Committees approved this consent procedure. This study was carried out in strict accordance with the recommendations in the Guide for the Care and Use of Laboratory Animals of Binzhou Medical University. The protocol was approved by the Committee on the Ethics of Animal Experiments of Binzhou Medical 
University. All procedures were performed under sodium pentobarbital anesthesia, and all efforts were made to minimize suffering of the animals.

\section{AUTHOR CONTRIBUTIONS}

ZL, TL, and JZ collected samples. XH and ZL conceived and designed the experiments as well as wrote the paper. ZL, MZ, and TL conducted the experiments. XH, ZL, MZ, TL, JZ, XL, YJ, and $\mathrm{HZ}$ analyzed the data and contributed reagents/materials/ analysis.

\section{REFERENCES}

1. Mahalakshmi B, Therese KL, Devipriya U, Pushpalatha V, Margarita S, Madhava HN. Infectious aetiology of congenital cataract based on TORCHES screening in a tertiary eye hospital in Chennai, Tamil Nadu, India. Indian J Med Res (2010) 131:559-64.

2. Li XL, Wei HX, Zhang H, Peng HJ, Lindsay DS. A meta analysis on risks of adverse pregnancy outcomes in Toxoplasma gondii infection. PLoS One (2014) 9(5):e97775. doi:10.1371/journal.pone.0097775

3. Jones JL, Lopez A, Wilson M, Schulkin J, Gibbs R. Congenital toxoplasmosis: a review. Obstet Gynecol Surv (2001) 56:296-305. doi:10.1097/ 00006254-200105000-00025

4. Mor G, Cardenas I, Abrahams V, Guller S. Inflammation and pregnancy: the role of the immune system at the implantation site. Ann N Y Acad Sci (2011) 1221:80-7. doi:10.1111/j.1749-6632.2010.05938.x

5. Faas MM, de Vos P. Uterine NK cells and macrophages in pregnancy. Placenta (2017) 56:44-52. doi:10.1016/j.placenta.2017.03.001

6. Mori M, Bogdan A, Balassa T, Csabai T, Szekeres-Bartho J. The decidual-the maternal bed embracing the embryo-maintains the pregnancy. Semin Immunopathol (2016) 38(6):635-49. doi:10.1007/s00281-016-0574-0

7. Trundley A, Gardner L, Northfield J, Chang C, Moffett A. Methods for isolation of cells from the human fetal-maternal interface. Methods Mol Med (2006) 122:109-22. doi:10.1385/1-59259-989-3:109

8. Ning F, Liu H, Lash GE. The role of decidual macrophages during normal and pathological pregnancy. Am J Reprod Immunol (2016) 75(3):298-309. doi:10.1111/aji.12477

9. Gordon S. Alternative activation of macrophages. Nat Rev Immunol (2003) 3:23-35. doi:10.1038/nri978

10. Goerdt S, Orfanos CE. Other functions, other genes: alternative activation of antigen-presenting cells. Immunity (1999) 10:137-42. doi:10.1016/ S1074-7613(00)80014-X

11. Svensson J, Jenmalm MC, Matussek A, Geffers R, Berg G, Ernerudh J. Macrophages at the fetal-maternal interface express markers of alternative activation and are induced by M-CSF and IL-10. J Immunol (2011) 187(7):3671-82. doi:10.4049/jimmunol.1100130

12. Nagamatsu T, Schust DJ. The contribution of macrophages to normal and pathological pregnancies. Am J Reprod Immunol (2010) 63(6):460-71. doi:10.1111/j.1600-0897.2010.00813.x

13. Cella M, Döhring C, Samaridis J, Dessing M, Brockhaus M, Lanzavecchia A, et al. A novel inhibitory receptor (ILT3) expressed on monocytes, macrophages, and dendritic cells involved in antigen processing. J Exp Med (1997) 185(10):1743-51. doi:10.1084/jem.185.10.1743

14. Castells MC, Wu X, Arm JP, Austen KF, Katz HR. Cloning of the gp49B gene of the immunoglobulin superfamily and demonstration that one of its two products is an early-expressed mast cell surface protein originally described as gp49. J Biol Chem (1994) 269(11):8393-401.

15. Ban YL, Kong BH, Qu X, Yang QF, Ma YY. BDCA-1+, BDCA-2+ and BDCA$3+$ dendritic cells in early human pregnancy decidua. Clin Exp Immunol (2008) 151(3):399-406. doi:10.1111/j.1365-2249.2007.03576.x

16. Rochat MK, Ege MJ, Plabst D, Steinle J, Bitter S, Braun-Fahrländer C, et al. Maternal vitamin D intake during pregnancy increases gene expression of ILT3 and ILT4 in cord blood. Clin Exp Allergy (2010) 40(5):786-94. doi:10.1111/j.1365-2222.2009.03428.x

\section{ACKNOWLEDGMENTS}

The authors would like to acknowledge the excellent technical assistance provided by the Medical Research Center Experiment Platform of Binzhou Medical University.

\section{FUNDING}

This work was supported by funds from the National Natural Science Foundation of China (grant numbers 81672049 and 81401687).

17. Matsumoto Y, Wang LL, Yokoyama WM, Aso T. Uterine macrophages express the gp49B inhibitory receptor in midgestation. J Immunol (2001) 166:781-6. doi:10.4049/jimmunol.166.2.781

18. Rojo S, Stebbins CC, Peterson ME, Dombrowicz D, Wagtmann N, Long EO. Natural killer cells and mast cells from gp49B null mutant mice are functional. Mol Cell Biol (2000) 20(19):7178-82. doi:10.1128/MCB.20.19. 7178-7182.2000

19. Gontijo da Silva M, Clare Vinaud M, de Castro AM. Prevalence of toxoplasmosis in pregnant women and vertical transmission of Toxoplasma gondii in patients from basic units of health from Gurupi, Tocantins, Brazil, from 2012 to 2014. PLoS One (2015) 10(11):e0141700. doi:10.1371/journal.pone.0141700

20. Remington JS, McLeod R, Desmonts G. Toxoplasmosis. 4th ed. In: Remington JS, Klein JO, editors. Infectious Diseases of the Fetus and Newborn Infant. Philadelphia, PA: The W. B. Saunders Company (1995). p. 140-267.

21. Liu X, Zhao M, Yang X, Han M, Xu X, Jiang Y, et al. Toxoplasma gondii infection of decidual $\mathrm{CD} 1 \mathrm{c}(+)$ dendritic cells enhances cytotoxicity of decidual natural killer cells. Inflammation (2014) 37(4):1261-70. doi:10.1007/ s10753-014-9853-x

22. Luppi P. How immune mechanisms are affected by pregnancy. Vaccine (2003) 21(24):3352-7. doi:10.1016/S0264-410X(03)00331-1

23. Liu Y, Zhao M, Xu X, Liu X, Zhang H, Jiang Y, et al. Adoptive transfer of Treg cells counters adverse effects of Toxoplasma gondii infection on pregnancy. J Infect Dis (2014) 210(9):1435-43. doi:10.1093/infdis/jiu265

24. Gustafsson C, Mjösberg J, Matussek A, Geffers R, Matthiesen L, Berg G, et al. Gene expression profiling of human decidual macrophages: evidence for immunosuppressive phenotype. PLoS One (2008) 3(4):e2078. doi:10.1371/ journal.pone.0002078

25. Svensson-Arvelund J, Ernerudh J. The role of macrophages in promoting and maintaining homeostasis at the fetal-maternal interface. Am J Reprod Immunol (2015) 74(2):100-9. doi:10.1111/aji.12357

26. Svensson-Arvelund J, Mehta RB, Lindau R, Mirrasekhian E, RodriguezMartinez H, Berg G, et al. The human fetal placenta promotes tolerance against the semiallogeneic fetus by inducing regulatory $\mathrm{T}$ cells and homeostatic M2 macrophages. J Immunol (2015) 194(4):1534-44. doi:10.4049/ jimmunol.1401536

27. Chang CC, Ciubotariu R, Manavalan JS, Yuan J, Colovai AI, Piazza F, et al. Tolerization of dendritic cells by $\mathrm{T}(\mathrm{S})$ cells: the crucial role of inhibitory receptors ILT3 and ILT4. Nat Immunol (2002) 3(3):237-43. doi:10.1038/ni760

28. Jin LP, Fan DX, Zhang T, Guo PF, Li DJ. The costimulatory signal upregulation is associated with Th1 bias at the maternal-fetal interface in human miscarriage. Am JReprod Immunol (2011) 66(4):270-8. doi:10.1111/ j.1600-0897.2011.00997.x

29. Nagamatsu T, Schust DJ. The immunomodulatory roles of macrophages at the maternal-fetal interface. Reprod Sci (2010) 17(3):209-18. doi:10.1177/ 1933719109349962

30. Abrahams VM, Kim YM, Straszewski SL, Romero R, Mor G. Macrophages and apoptotic cell clearance during pregnancy. Am J Reprod Immunol (2004) 51(4):275-82. doi:10.1111/j.1600-0897.2004.00156.x

31. Breburda EE, Dambaeva SV, Slukvin II, Golos TG. Selective distribution and pregnancy-specific expression of DC-SIGN at the maternal-fetal interface in the rhesus macaque: DC-SIGN is a putative marker of the recognition of pregnancy. Placenta (2006) 27(1):11-21. doi:10.1016/j. placenta.2004.11.006 
32. Kong L, Zhang Q, Chao J, Wen H, Zhang Y, Chen H, et al. Polarization of macrophages induced by Toxoplasma gondii and its impact on abnormal pregnancy in rats. Acta Trop (2015) 143:1-7. doi:10.1016/j.actatropica.2014. 12.001

33. Hibbs JB Jr, Vavrin Z, Taintor RR. L-arginine is required for expression of the activated macrophage effector mechanism causing selective metabolic inhibition in target cells. J Immunol (1987) 138(2):550-65.

34. Yang Z, Ming X. Functions of arginase isoforms in macrophage inflammatory responses: impact on cardiovascular diseases and metabolic disorders. Front Immunol (2014) 5:533. doi:10.3389/fimmu.2014.00533

35. Neri I, Mazza V, Galassi MC, Volpe A, Facchinetti F. Effects of L-arginine on utero-placental circulation in growth-retarded fetuses. Acta Obstet Gynecol Scand (1996) 75:208-12. doi:10.3109/00016349609047088

36. Wink DA, Hines HB, Cheng RY, Switzer CH, Flores-Santana W, Vitek MP, et al. Nitric oxide and redox mechanisms in the immune response. J Leukoc Biol (2011) 89(6):873-91. doi:10.1189/jlb.1010550

37. Garvey EP, Tuttle JV, Covington K, Merrill BM, Wood ER, Baylis SA, et al. Purification and characterization of the constitutive nitric oxide synthase from human placenta. Arch Biochem Biophys (1994) 311(2):235-41. doi:10.1006/ abbi.1994.1232

38. Wang X, Wang J, Trudinger B. Gene expression of nitric oxide synthase by human umbilical vein endothelial cells: the effect of fetal plasma from pregnancy with umbilical placental vascular disease. BJOG (2003) 110(1):53-8. doi:10.1046/j.1471-0528.2003.01329.x
39. Zhao M, Zhang R, Xu X, Liu Y, Zhang H, Zhai X, et al. IL-10 reduces levels of apoptosis in Toxoplasma gondii infected trophoblasts. PLoS One (2013) 8(2):e56455. doi:10.1371/journal.pone.0056455

40. Zhang R, Zhang H, Liu X, Fu Q, Xu X, Hu X. The immunoprotective role of interleukin-10 in abnormal pregnancy outcome induced by Toxoplasma gondii infection. Gynecol Obstet Invest (2012) 73(3):223-9. doi:10.1159/000333310

41. Hunt JS. Macrophages in human uteroplacental tissues: a review. Am J Reprod Immunol (1989) 21:119-22. doi:10.1111/j.1600-0897.1989.tb01015.x

42. Yui J, Hemmings D, Garcia-Lloret M, Guilbert LJ. Expression of the human p55 and p75 tumor necrosis factor receptors in primary villous trophoblasts and their role in cytotoxic signal transduction. Biol Reprod (1996) 55:400-9. doi:10.1095/biolreprod55.2.400

Conflict of Interest Statement: The authors declare that the research was conducted in the absence of any commercial or financial relationships that could be construed as a potential conflict of interest.

Copyright (C) 2017 Li, Zhao, Li, Zheng, Liu, Jiang, Zhang and Hu. This is an openaccess article distributed under the terms of the Creative Commons Attribution License (CC BY). The use, distribution or reproduction in other forums is permitted, provided the original author(s) or licensor are credited and that the original publication in this journal is cited, in accordance with accepted academic practice. No use, distribution or reproduction is permitted which does not comply with these terms. 